\title{
Tailored hypersound generation in single plasmonic
}

\section{nanoantennas}

Fabricio Della Picca ${ }^{1 \dagger}$, Rodrigo Berte ${ }^{2,3 \dagger}$, Mohsen Rahmani ${ }^{2 \ddagger}$, Pablo Albella ${ }^{2}$, Juan M. Bujjamer ${ }^{1}$, Martín Poblet $^{1}$, Emiliano Cortés ${ }^{2}$, Stefan A. Maier ${ }^{2 *}$, Andrea V. Bragas ${ }^{1 *}$

1- Laboratorio de Electrónica Cuántica, Dep. de Física, FCEN_IFIBA CONICET, Universidad de Buenos Aires, Intendente Güiraldes 2160, C1428EGA, Buenos Aires, Argentina

2- The Blackett Laboratory, Department of Physics, Imperial College London, London SW7 2AZ, United Kingdom

3- CAPES Foundation, Ministry of Education of Brazil, Brasilia - DF 70040-020, Brazil

KEYWORDS: coherent acoustic phonon, nanoantenna, nanoresonator, hypersound, pump-probe

ABSTRACT: Ultra-short laser pulses impinging on a plasmonic nanostructure trigger a highly dynamic scenario in the interplay of electronic relaxation with lattice vibrations, which can be experimentally probed via the generation of coherent phonons. In this letter we present studies of hypersound generation in the range of a few to tens of GHz on single gold plasmonic nanoantennas, which have additionally been subjected to pre-designed mechanical constraints via silica bridges. Using these hybrid gold/silica nanoantennas, we demonstrate experimentally and via numerical simulations how mechanical constraints allow control over their vibrational mode spectrum. Degenerate pump-probe techniques with double 
modulation are performed in order to detect the small changes produced in the probe transmission by the mechanical oscillations of these single nanoantennas.

Nanometric control of shape, size, composition and interaction of plasmonic nanoantennas has become a valuable design tool for the creation of a tailored optoelectronic response. These parameters also determine the mode spectrum of their individual and collective mechanical vibrations, which have a fundamental importance for the understanding of electronic relaxation processes to the crystal lattice and the ensuing propagation of sound and heat at the nanoscale. Indeed, the generation of hypersonic waves in a variety of materials, geometries and devices, has grown to applications in different areas such as high-frequency electronics [1-3], biomedical science [4, 5], ultrasensitive mass detection [6,7] and photoacoustic imaging $[8,9]$. Whereas the generation of coherent vibrations in metal nanoparticles [10-13], or in small groups of them $[14,15]$ has been subject of active research during the last decade, their applications as local and tunable nanoresonators are still in their beginnings [8].

Besides these applications, experiments of generation of coherent phonons with ultrashort laser pulses also reveal detailed information about fundamental dynamical processes occurring in solids. Techniques for detection and control of coherent optical and acoustic phonons have been intensively studied, as well as the various physical underlying generation mechanisms discussed [16-21]. In plasmonic nanostructures, light pulse absorption generates a population of hot electrons which can be further enhanced by the resonant excitation of surface plasmons. The electrons relax through Landau damping within the first femtoseconds and then through electron-electron scattering on the hundreds of femtoseconds to picosecond scale [22], followed by thermal equilibration with the lattice ions, hence launching mechanical 
oscillations of the nanostructure at its normal modes frequencies. Those oscillations, known as coherent acoustic phonons, produce periodic perturbations in the dielectric constant which can be detected as a transmission change of a probe pulse. For frequency modes on the order of few tens of picoseconds, the main excitation mechanism comes from the lattice dilation produced by the fast heating of the lattice with a concomitant change in the oscillator equilibrium position [23].

Plasmonic relaxation in metallic nanoantennas leads in general to the simultaneous launching of all normal mode oscillations compatible with a symmetric strain profile, without the possibility of a selective generation of a given mode. Selective excitation however requires indeed controlled manipulation of the shape and symmetry of the nanostructure. However, selectivity on the detection of a subset of modes is possible to some extent by performing spectrally [15] - and/or polarization - resolved measurements of probe transmission [24]. Using selective polarization probe detection, O'Brien et al [24] recently measured symmetric and antisymmetric modes in a gold Swiss-cross, and performed temporal coherent control using two delayed pump pulses in order to enhance or suppress one given phonon mode. Additionally, Chang et al [25] studied the influence of the adhesion layer thickness in the acoustic frequency value of single gold nanodisks, obtaining in this way a wide tunability of the in-plane breathing mode.

In this letter, we performed a different approach introducing metal nanoantennas with mechanical constraints that modify the phonon spectra, without appreciably changing the plasmonic response. Mechanical constraints modify the symmetry of the system and as a consequence alter the amount of power effectively delivered to each one of the different acoustic modes. Although the thermal excitation is distributed isotropically due to the fast plasmon decay, the mechanical constraints allow only certain modes to develop, favoring some of them and lowering others. We present in this work four different designs of gold nanoantennas with silica mechanical constraints fabricated by a two-step electron beam 
lithography process, and we show the generation of coherent phonons in each of these single nanooscillators in the range of tens of GHz.

Briefly, using electron-beam lithography we have fabricated Au nanorod antennas on a quartz substrate, using a two nanometer thick $\mathrm{Cr}$ adhesion layer. In a second lithographic step we have positioned, with an accuracy of below ten nanometers, hydrogen silsesquioxane (HSQ) patches at defined locations with respect to the antennas (see Figure 1). Once exposed to the electron beam, HSQ's chemical and optical properties become comparable to $\mathrm{SiO}_{2}$ (see supplementary information for fabrication details). Using this approach of fabrication of hybrid gold/silica nanoresonators, we can change the normal modes of gold nanoantenna, with or without appreciably affecting the plasmonic properties, depending on the position of the silica patches. These patches restrict the expansion of the underlying nanoantennas upon pulsed laser excitation, and consequently alter the vibrational mode spectrum, as we will show in the following.

Here we have compared the phononic response of four different nanoantennas: naked-rod, center-silica, ends-silica and all-silica, as shown in Figure 1a)-b). The naked-rod plasmon resonance around 800nm, Figure $1 \mathrm{c})-\mathrm{d}$ ), is nearly not disturbed due to the presence of the silica in the center-silica design, since it is grown far from the highest electronic density located at the tips of the rod for dipolar excitation. On the other hand, for the other two geometries the plasmonic resonance shifts slightly (ends-silica) or stronger (all-silica) to the red, as shown in Figures 1c)-d). 
a)

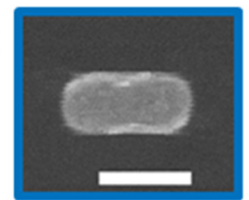

b)

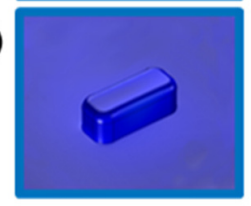

c)
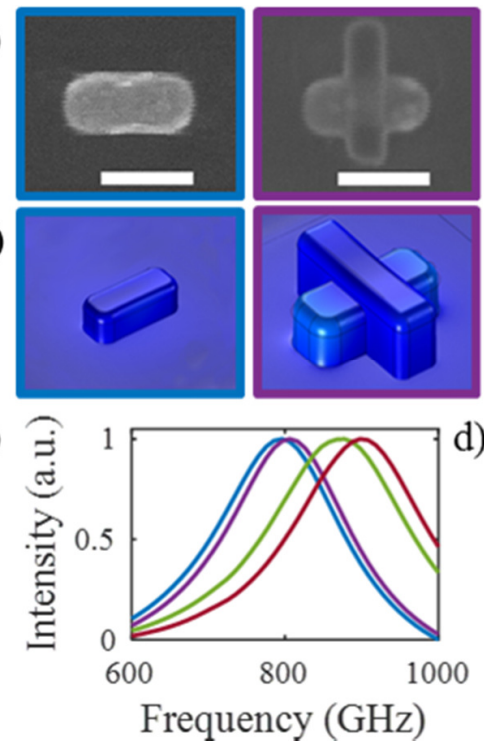

d)
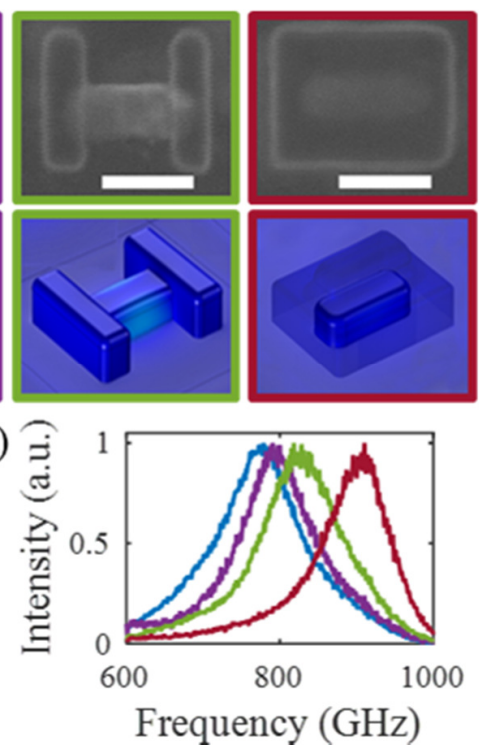

e)
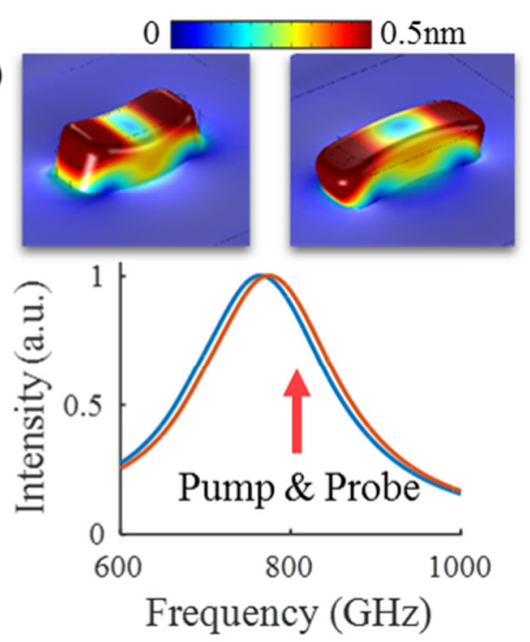

Figure 1. Mechanically constrained nanoantennas as localized acoustic resonators. A layer of silica is selectively positioned on top of a gold nanorod, modifying separately the mechanical and plasmonic responses. a) Scanning Electron Microscopy (SEM) images of the gold nanoantennas grown on a quartz substrate by two step e-beam lithography: naked-rod, center-silica, ends-silica and all-silica, from left to right. Scale bars correspond to $100 \mathrm{~nm}$. b) 3D designs used for the finite element method (FEM) simulations in COMSOL Multiphysics ${ }^{\mathrm{TM}}$ software. Simulated dimensions were extracted from SEM and Atomic Force Microscopy (AFM) images on the samples. c) - d) Simulated and experimental scattering spectra of the four types of designs, respectively. In both cases: blue curves correspond to the naked-rod; purple ones to the centered-silica; green for the ends-silica and red for all-silica. e) Top panel shows the total displacement maps (20x scale factor applied to highlight mode deformation) of a naked-rod at two different phase values of its extensional oscillation mode, induced after the pump pulse arrival. Bottom panel represents schematically the change of the optical response produced by nanorod contraction and expansion. (The size of the nanorod was taken into account, considering that the total volume of the structure must remain the same).

Generation of coherent acoustic phonons was conducted with a degenerate pump-probe technique using double (pump and probe) modulation and lockin detection at the difference frequency (see supplementary information, experimental set-up). The output beam of a Ti:Sapphire laser with $95 \mathrm{MHz}$ of repetition rate, $100 \mathrm{fs}$ pulses set at $800 \mathrm{~nm}$ wavelength was focused onto a single nanoantenna with a pump (probe) spot 
size diameter of $2.5 \mu \mathrm{m}(1 \mu \mathrm{m})$, using a home-made microscope. The average pump power at the sample was $3 \mathrm{~mW}$ and the probe power $0.1 \mathrm{~mW}$.

To further support and interpret experimental results on single nanoantennas, numerical simulations were performed to assess the mechanical response of each one of the different geometries shown in Figures $1 b$ ). Finite element methods for linear elastic theory have been established as a powerful tool to validate and predict the acoustic response of objects extending from a few nanometers in size to those of macroscopic dimensions [23]. Indeed, modeling based on the solution of Navier's equation of continuum mechanics shows excellent agreement with experimental results, diverging from atomistic method predictions only for objects comprising a few hundreds of atoms [23, 26]. Although nanomaterials are widely studied due to unique properties arising at this size regime, the invariance of some of the physical properties of nanosized materials compared to their bulk counterparts, for instance the Young's modulus for gold nanowires [27], justifies a macroscopic approach when applied to such a small size regime [23]. Conversely, an increase in the yield strength of gold for nanometric wires makes these structures more resilient to mechanical stress [27], which may suggest that higher limits for the lattice dilation can be achieved without inducing a plastic deformation on the rods. Indeed, we did not observed structural alterations in any of the investigated geometries after the performed pump-probe experiments for the laser power and repetition rate applied. We reiterate that a combination of SEM and AFM characterization has been performed to determine the proper representative dimensions of the gold nanorods and each silica structure to model the different cases (see supplementary figure 1 for the AFM profiles).

In order to understand the information that can be extracted from coherent acoustic measurements of our nanostructures, let us first analyze the classical equation of motion of the oscillation part, describing the coherent phonon field with frequency $\omega_{q}$, for small nuclear displacements: 


$$
\mu\left[\frac{\partial^{2} q(t)}{\partial t}+2 \gamma \frac{\partial q(t)}{\partial t}+\omega_{q}^{2} q(t)\right]=F(t)
$$

for a driven damped oscillator with reduced lattice mass $\mu$ and damping $\gamma . \mathrm{F}(\mathrm{t})$ is the driving force which is defined by the specific light-matter interaction. It is well known $[19,23]$ that for metal nanostructures the driving force has two main contributions, one from the electron and the other from the lattice heating. After the pump pulse arrives, the hot electrons couple directly to the lattice by the deformation potential and provide a fast impulsive force $\mathrm{F}^{\mathrm{e}}(\mathrm{t}) \propto \mathrm{e}^{-\mathrm{t} / \tau_{\mathrm{ep}}}$, decaying at the electron-phonon coupling time $\tau_{e p}$. The second mechanism is indirect and displacive in nature, originating from a dilation of the lattice and change of the initial equilibrium position, due to the anharmonicity of the lattice potential. This leads to a driving force $\mathrm{F}^{\mathrm{l}}(\mathrm{t}) \propto\left(1-\mathrm{e}^{-\mathrm{t} / \tau_{\mathrm{ep}}}\right)$. Both contributions are always present, but their amplitude ratio defines the initial phase of the solution of Eq. (1). For short ( 100fs) excitation pulse-width and periods on the tens of picosecond, the dominant mechanism is the displacive one, ending up in a cosine-type oscillation $[13,19,23]$. However, the phase is the most sensitive of the parameters to test either the underlying mechanisms or any coupling between modes [20], at times making interpretation difficult [24, 28].

Top panel of the Figure 1e) shows the resulting 3D displacement maps for the lowest frequency mode sustained by the naked-rod, at two different phase values. Here, a 20x scale factor was applied to all components of the displacement to emphasize the small contraction/expansion of the mode. The color scale bar represents the actual (i.e. not scaled) sub-nanometer displacement values obtained for the estimated $100 \mathrm{~K}$ increase in lattice temperature. It is an extensional mode although it embraces a tridimensional movement (full 3D simulation videos can be found in the supplementary information). As the particle expands and contracts, the plasmon resonance is modulated back and forth at the frequency $f_{q}=\omega_{q} / 2 \pi$, producing changes in the probe transmission $T$ at this same frequency. If now several (uncoupled) modes contribute to those changes at the same time, then: 


$$
\frac{\Delta T}{T}=\sum_{q} A_{q} e^{-\gamma_{q} t} \cos \left(2 \pi f_{q} t+\varphi_{q}\right)
$$

where the amplitude $A_{q}$ is dependent on the probe polarization. In all our experiments the pump and probe polarizations are set parallel to the long-axis of the rod (x-direction), so that $A_{q}$ will reveal those tridimensional displacements which modify the amplitude, width and/or peak frequency of the longitudinal plasmon resonance.
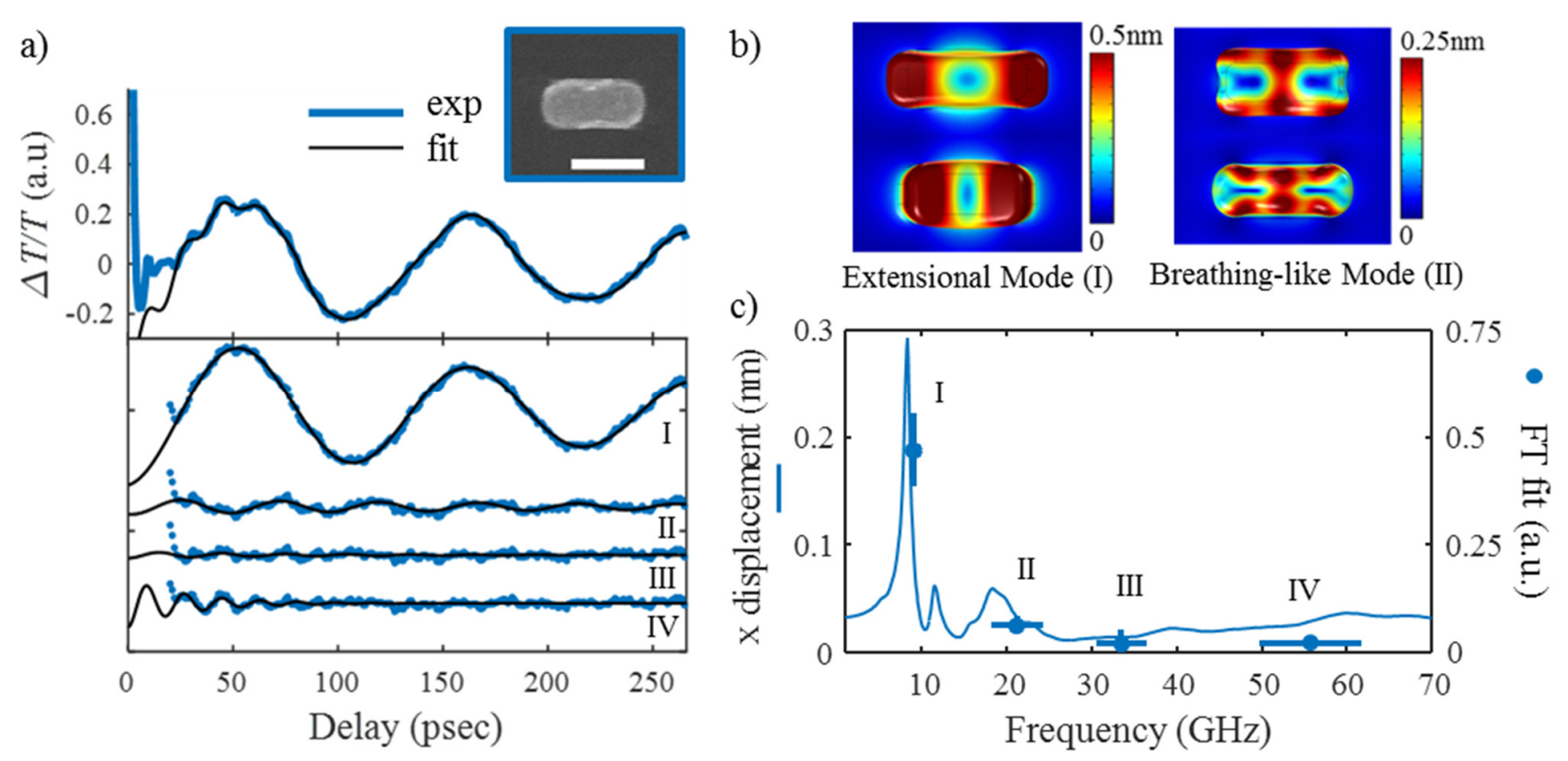

Figure 2. Coherent acoustic phonons in the naked-rod nanoantenna. a) On top, the experimental total differential probe transmission as a function of the pump-probe delay (exponential decay subtracted) and at the bottom the decomposition into the four modes detected (see main text, vertically shifted for clarity). Time traces are the intensity of the probe transmitted light at the difference between the pump and probe frequency modulations. The inset shows the SEM image of the naked-rod nanoantenna tested. Scale: $100 \mathrm{~nm}$. Pump and probe wavelength at $800 \mathrm{~nm}$ and both polarizations set parallel to the $\mathrm{x}$-axis (long-axis of the rod direction). b) Top view of the 3D simulation for the two lowest frequency modes, the extensional (I) and the breathing-like (II). The snapshots are taken at the instants of highest expansion and contraction. 20x and 40x deformation scale factors were applied to modes I and II, respectively. Full 3D simulation videos can be found in the supplementary information. c) Simulated eigenmode spectrum shows the average amplitude for the displacement in x-direction obtained for the rod domain as a function of strain frequency. The dots are the amplitudes of the Fourier transform of the fits of the experimental traces in a) 
and the error bar contains the parameter error as well as the frequency and amplitude measured in other single nanoantennas of the same type.

The analysis of the data obtained for the naked-rod nanoantenna is shown in Figure 2, and will serve as a comparison to the other acoustic nanoresonators. Temporal traces are expected to contain a sum of damped sinusoidals as in Eq. (2) and as shown in Figure 2 a). Fitting of the experimental data has been performed in two steps. First, approximating the four parameters $A$ (amplitude), $f$ (frequency), $\gamma$ (damping) and $\varphi$ (phase), with linear prediction methods (for details on the analysis, see supplementary information) to first narrow the parameter range, and then using those values as initial parameters for the non-linear fit, obtaining high confidence intervals (see Table 1, and supplementary Table 1 with the complete set of fitting parameters). There are four modes detected at 9.1 (I), 21 (II), 34 (III) and 56 (IV) GHz, among which I (extensional) and II (breathing-like) are the highest in amplitude and lowest in damping. Top panel of Figure 2 a) shows the time trace with the fit superimposed, whereas the bottom panel shows the individual mode contributions, obtained as subtraction of the fits of each one of the other modes from the total time trace. Top-views of the simulation for the extensional and breathing-like modes are shown in Figure $2 \mathrm{~b}$ ), where the color maps indicate the magnitude of the displacement (see also supplementary videos 1 and 2 for the 3D displacements of both modes). Clearly, both modes produce periodic changes in the probe transmission polarized in the $\mathrm{x}$ direction, due to the differential changes in length and aspect ratio which, in turn, produce the oscillation of the longitudinal plasmon resonance. Since the probe wavelength is set at the red-side of the plasmon resonance, Figure $1 \mathrm{e}$ ), and a displacive driving force is expected, a cosine-like oscillation with $\pi$ initial phase is predicted, due to the red shift of the longitudinal plasmon resonance with length increase [29]. The simulated spectral response of the x-displacement due to a frequency dependent thermal strain can be appreciated in Figure 2c) with the amplitudes of the Fourier transform of the fits of the experimental traces superimposed as full dots. Error bars account for the 
parameter fitting error and also for the frequency dispersion obtained from measurements taken on different single structures. Considering these error bars, the agreement between experiment and simulation is fairly good, only some differences deserve to be discussed next.

In the high frequency zone of the spectrum, III and IV, it is difficult to match the experimental measurements with the simulated profile, due to the large number of modes with low amplitude and high damping shown by the simulations. Those modes are complex distributions of nodes and peaks. The experiment selects among them those which have total 3D displacements that affect the probe transmission; whereas the simulations only show the average $\mathrm{x}$-displacement amplitude. In the following we will only discuss modes I and II, to compare experiment with simulations, but having in mind that the high frequency modes are detectable.

The influence of the underlying adhesion layer in the frequency and damping of the acoustic modes has been discussed extensively by Chang et al [25] and used as a means for tuning the fundamental mode frequency by about $25 \%$. In our simulations we assume that the elastic properties of gold are those of the bulk material [27] and we also included the $2 \mathrm{~nm}$ chromium adhesion layer used in the fabrication. The resulting extensional mode for this configuration (attached to a quartz substrate) lies between those obtained for a free boundary condition, that gives a lower frequency resonance, or a fixed bottom that overestimates it (supplementary figure 2 a). It has been shown [25] that increasing the adhesion layer thickness leads to a stronger binding to the substrate. We obtained the same results in our case through the simulations of the frequency of mode I as a function of chromium layer thickness (supplementary figure 2 a). For these reasons, all calculated frequencies are very sensitive to the elastic properties of the boundary between gold and the substrate. Additionally, the small peak that appears just on the high frequency side of mode I has not been detected experimentally. This peak should be a consequence of the 
presence of the substrate and is the result of the coupling between the in-plane and out-of-plane modes (supplementary figure 2b) and has been observed in a similar situation in the literature [24].

a)
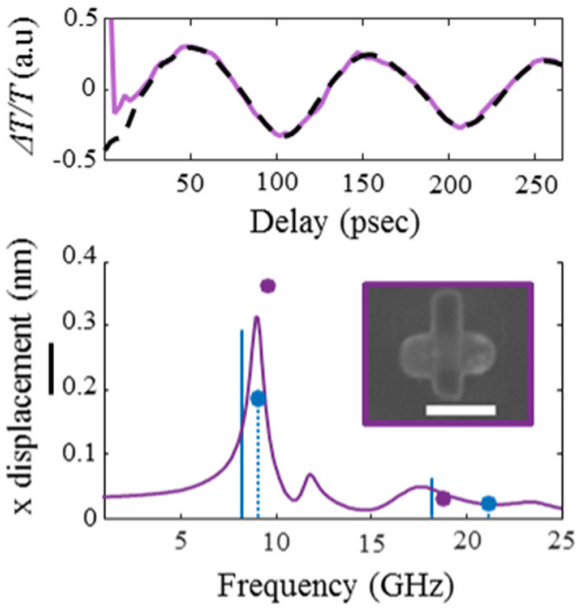

b)
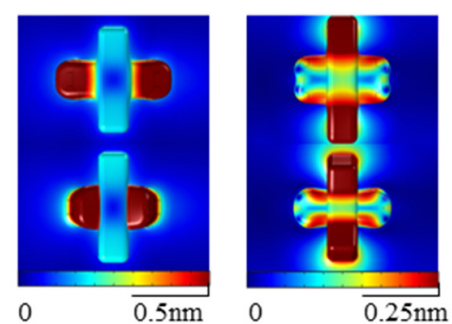

c)
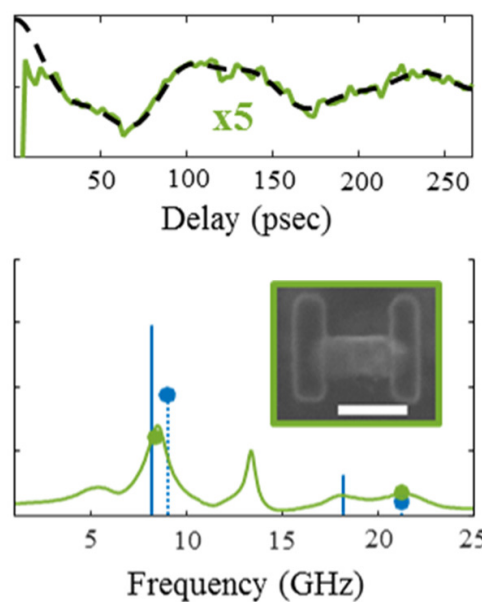

d)

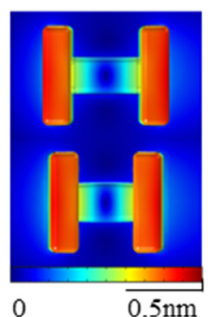

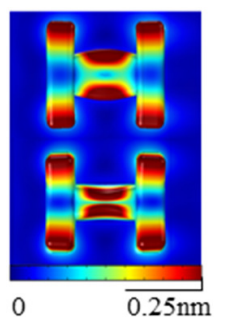

e)
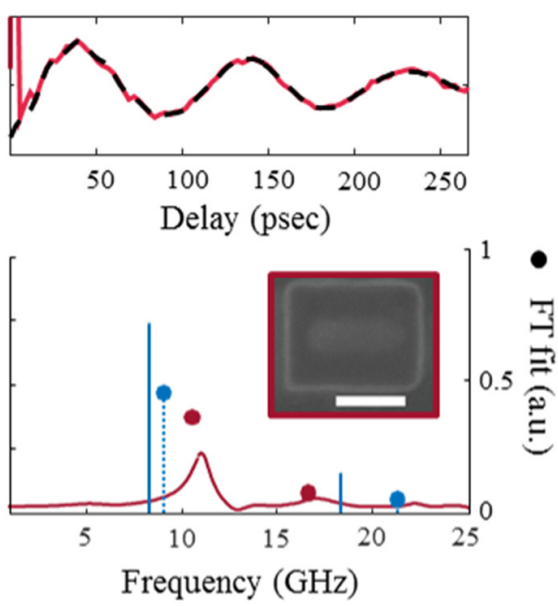

f)

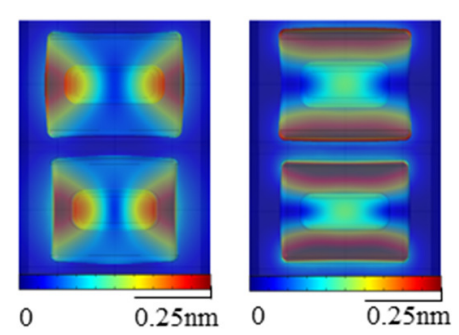

Figure 3. Comparative analysis of constrained antennas with the naked-rod spectrum. Experimental results and simulation of modes I (extensional) and II (breathing-like) for the center-silica (a-b), endssilica (c-d) and all-silica (e-f) geometries, respectively. Color maps show the magnitude of the displacement (deformation scale factors are used to highlight the shape changes, see supplementary information 5). For each one of the cases, the pump probe signal, $\Delta T / T$, is shown at the top (black dashed lines are the fits), and at the bottom the vibrational spectra compared with the naked-rod case (light blue line and dots, for simulated and experimental points, respectively). Left scale of the vibrational spectra in a), c) and e) corresponds to the simulated eigenmode spectrum in nm, whereas the right scale shows the amplitude of the Fourier transform of the fits of the experimental traces, in the same arbitrary scale as in Figure 2. SEM images are shown as insets, scale: 100nm. In b), d) and f) the left (right) panel shows the extensional (breathing-like) mode. See text for the discussion. Note that continuity of stress and displacement between nanorods and silica patches in all simulations, leads in some cases, to larger displacements for the silica than for the nanorod itself, due to the different elastic moduli. 
Table 1. Parameters extracted from the fitting of the experimental pump-probe measurements. Names defined in Eq (2). Error of the parameters at the last digit. The first (second) place in each cell of the table corresponds to the mode I (II).

\begin{tabular}{|c|cc|cc|cc|cc|cc|}
\hline & \multicolumn{2}{|c|}{$\boldsymbol{f}(\mathbf{G H z})$} & \multicolumn{2}{c|}{$\boldsymbol{A}$} & \multicolumn{2}{c|}{$\boldsymbol{\gamma}(\mathbf{1} / \mathbf{n s e g})$} & \multicolumn{2}{c|}{$\boldsymbol{\varphi ( \pi )}$} & \multicolumn{2}{c|}{$\boldsymbol{Q}$} \\
\hline Naked & 9.1 & 21 & 0.31 & 0.03 & 3.3 & 3.7 & 1.03 & 0.93 & 8.6 & 18 \\
\hline Center & 9.6 & 19 & 0.36 & 0.04 & 2 & 4.2 & 1.02 & 1 & 15 & 14 \\
\hline Ends & 8.5 & 21 & 0.08 & 0.02 & 6.5 & 6.4 & 2 & 1.92 & 4.1 & 10 \\
\hline All & 10.5 & 17 & 0.36 & 0.04 & 4.8 & 6.3 & 1.12 & 1.15 & 6.9 & 8.3 \\
\hline
\end{tabular}

Next, we present a comparative analysis between the different nanoresonators and the naked-rod one, shown in Figure 3 and Table 1. All along Figure 3, the frequency values for the naked-rod are superimposed for comparison, as light blue lines for the simulated values and as light blue dotted lines and dots for the experimental values. Table 1 shows the parameters defined in Equation (2), extracted from the fitting of the experimental time traces. We start our discussion with the center-silica design shown Figures 3a) and 3b). There is a good agreement between experimental and simulated frequencies I and II, marked as purple dots and lines in that Figure, respectively. As shown in the left panel of Figure 3b), the silica layer is located at the node of the extensional mode I and so, only small changes in the frequency and amplitude of this mode are expected, with respect to the naked-rod case. Indeed, the experimental signal appears neater since the silica favors thermal dissipation $[8,30]$, and the signal to noise increases as well as the amount of laser intensity that can be delivered to the nanoantenna without damaging the sample. The experimental extensional frequency $f_{\text {ext }}$ moves to a $5 \%$ higher value, suggesting that the silica layer strengthens the binding of the nanoantenna to the substrate and increases the restoring force $[25,31]$. Additionally, the quality factor $Q_{e x t}=\frac{\pi f_{e x t}}{\gamma}$ increases from 8.6 to 15 (Table 1), which makes it appear as a higher peak in the experimental FT value (Figure 3a)), although similar amplitude in the time trace is obtained. This reduction of the total damping has to be related to one or both of the two main 
contributions: the internal material damping and/or the acoustic radiation damping. Important to note is that inhomogeneous broadening due to morphological differences among a population of nanoantennas is absent because we measured the response of single rods. The internal material damping that accounts for the gold internal friction, inhomogeneities and disorder-related effects [32] should be similar in both situations (i.e. with and without silica added) and therefore may not be the reason for the quality factor change. On the other hand, the acoustic radiation damping is dictated by the acoustic mismatch between the impedances $Z=\rho v_{L}$ of the gold and the environment, where $\rho$ is the density of the material and $v_{L}$ is the longitudinal speed of sound. For the naked-rod case, the main source of acoustic losses is through the substrate, since the gold/air impedance mismatch is huge [33] and, as a result, the generated hypersound does not propagate in air. As already mentioned, for the center-silica nanoantenna the silica layer is added in a region where the displacement produced by the extensional mode is minimum and as a result the silica itself does not move much (Figure 3b and supplementary videos for 3D displacements). In other words, the silica does not add a new damping channel whereas at the same time it increases the binding to the substrate, lowering the losses produced by defects in interface quality. As a result, the total damping decreases and $Q_{\text {ext }}$ increases. For the breathing-like mode II of frequency $f_{b}$, on the contrary, the silica does affect the acoustic radiation damping, changing $Q_{b}=\frac{\pi f_{b}}{\gamma}$ from 18 to 14 . As can be seen from the right panel in Figure $3 \mathrm{~b}$ ), the silica moves in the direction perpendicular to $\mathrm{x}$, and also produces the lowering of $f_{b}$ by about $10 \%$ due to a higher effective mass.

We now move to the ends-silica design, shown in Figure 3c) and d). As before both simulations and experiment show good agreement in the values of the frequencies of the extensional and breathing-like modes (green lines and dots). These frequencies are now similar to those of the naked-rod, but a marked decrease in the amplitude of the extensional mode is evident (signals are multiplied by 5), since silica 
layers tend to anchor the edges, reducing the displacement in the $\mathrm{x}$ direction (see supplementary videos) and at the same time lowering the frequency due to the increased mass. In this case, the existence of the two modes are clearly seen even from the time trace shown in Figure 3c, where now the breathing-like oscillation shows up markedly, making the curve more squared. This is thus an example of how strongly the vibrational response can be modified, by changing the relative amplitude between the modes and favoring the appearance of one of them. In this configuration the plasmonic response is also affected (Figure 1 c) and d)), since the silica is located in the higher electric field region produced by the longitudinal plasmon, resulting in a spectral red shift. The probe wavelength is now at the blue-side of the absorption spectrum and as a consequence the coherent phonon signal is out of phase ( $\pi$ change, or $2 \pi$ initial phase, see also Table 1) with respect to the signal of the naked-rod and center-silica [13, 34, 35].

Finally, the all-silica design is the one which produces the highest changes in both extensional and the breathing-like frequencies, as can be seen in Figure 3 e) and f). $f_{\text {ext }}$ is increased by $15 \%$, whereas $f_{b}$ is decreased by $20 \%$. In the latter case, the reduction may be due to the higher mass that the breathing mode has to move, since the amount of silica that is participating in the oscillation is greater in this mode than in the extensional one, as can be seen from Figure $3 \mathrm{f}$ ) and also from the supplementary videos. At the same time, this effect is competing with the better adhesion of the rod to the substrate, which, as has been already demonstrated, results in an increment of the mode frequency in the case of the extensional mode. Comparing this observation with the extensional mode of the ends-silica case we observe an opposite behavior, since silica clamps much more efficiently the structure in the all-silica than in the ends-silica case. We also note that the phase of this time trace is different from the previous two examples (see Table 1). This sample is excited far from the plasmon resonance (see Figure 1c) and d)), and so, a more impulsive behavior should be expected, i.e, closer to a sine-like behavior ( $0.5 \pi$ phase and uneven multiples). However, the phase takes the value $1.12 \pi$, which is neither impulsive nor displacive. As has been already 
mentioned, this is the most sensitive parameter that accounts for the generation mechanisms and the coupling between modes; clearly the observed phase effects merit further investigations.

As a summary, we show in Figure 4 the acoustic frequencies (a) and amplitude ratios between modes I and II (b), for the four types of nanoresonators. The overall agreement of the experimental and simulated frequencies is very good, while the amplitude ratios show some discrepancies, although the general tendency shows the same behavior. This is understandable since, as we already mentioned, the simulations account for the displacement in $\mathrm{x}$ only, whereas the full complex 3D movement contributes in a non-trivial way to the total amplitude of the differential probe transmission. Also, it is important to bear in mind that the simulations performed do not consider possible structural defects and crystalline domain distributions existing in the rods, which might change its phononic response. The anisotropy of the elastic response of a crystal is usually neglected in simulations due to more pronounced effects of size and geometry dispersions of the corresponding measured ensemble on the particular modes (see supplementary figure 3a). Thus, the use of statistically-averaged Young's modulus and Poisson's ratio of randomly oriented crystalline domains when modelling might also be a source of error when comparing to measurements performed in single nanostructures [23]. Supplementary table 2 includes all the properties of materials used for our FEM numerical simulations. Therefore, in general terms, we can say that the experimental values of ratios and amplitudes modulate much more widely than the values predicted by the simulations. 

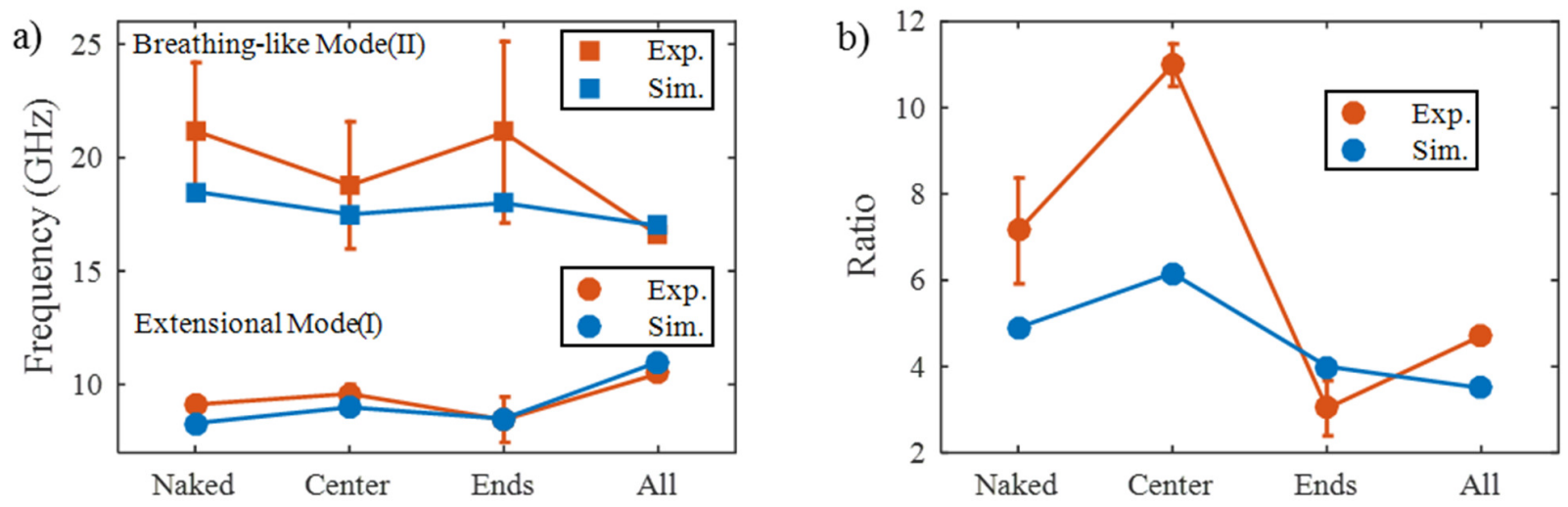

Figure 4. Variations in resonance frequencies and amplitudes extracted from measurements and simulations a) Frequencies of the lowest modes, extensional mode I and breathing-like mode II, for each of the designs. There is a total modulation of the extensional (breathing) frequency of about $30 \%$ (20\%). b) Amplitude ratio between extensional and breathing frequencies for the four nanoresonators. Experimental values show a markedly higher modulation than the simulated ones.

The examples introduced here serve as a proof of concept of a wider range of possibilities of manipulation of the phononic response of a plasmonic nanoantenna via space-selective deposition of oxide constraint patches. Following these ideas one can envision broad tunability and fine selection of modes of the hypersonic acoustic waves generated by these types of hybrid nanaoantennas. As a final remark, we would further like to stress the potential of this method by showing an example of a simple way to tune widely the low frequency mode, for the case of the end-silica design. By merely changing the height of the silica between 40 and $80 \mathrm{~nm}$, simulations show that the frequency changes by more than $100 \%$. The overall mode shift is attributed to an increasing proportion of the silica mass in the total mass of the nanoresonator, for which now the system oscillatory behavior is dominated by the geometrical parameters of the patches instead of those of the rod. Additionally, this tuning of the phononic response does not alter the plasmonic response of the nanoantenna. This substantiates that a change of the phononic response can be rather independent of the plasmonic response. 
a)

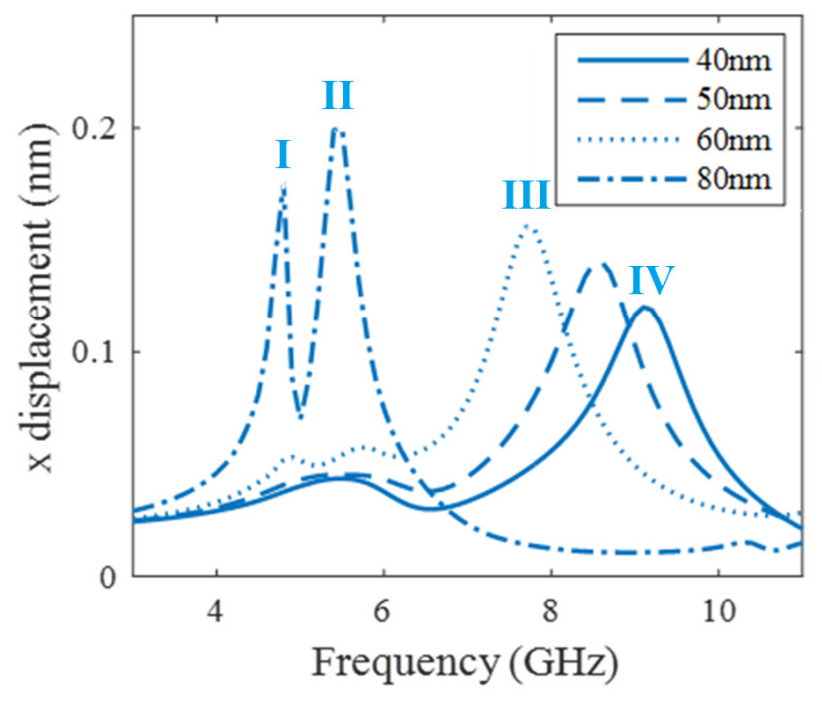

b)

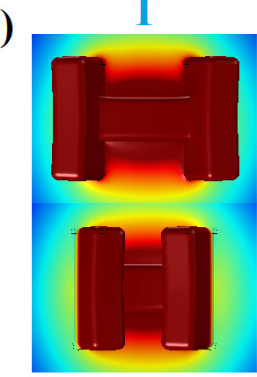

d)

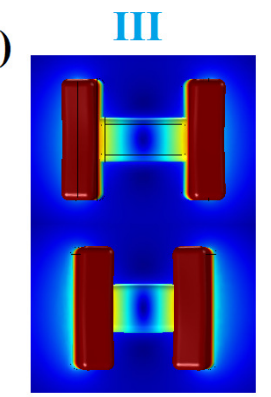

c)

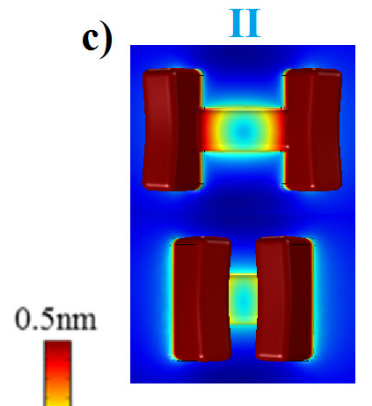

e)

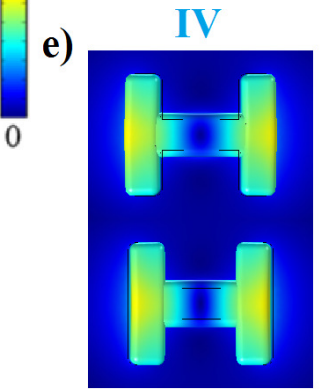

Figure 5: Numerical simulations of the silica height sweep vs. frequency in the case of the ends-silica nanoresonator. a) X displacement amplitude for different silica thicknesses. The low frequency mode shows a wide tunability of more than $100 \%$, just touching by little the silica height. (b-e) Top view of selected modes (I-IV) for the following constrains: b) $80 \mathrm{~nm}$ silica (I), c) $80 \mathrm{~nm}$ silica (II), d) $60 \mathrm{~nm}$ silica (III) and e) $40 \mathrm{~nm}$ silica (IV). Modes II, III and IV are similar to the extensional mode obtained for a $50 \mathrm{~nm}$ silica patch, the latter shown on the left panel of Figure 3d. A higher substrate displacement is evident on mode I, which contains a larger out of plane component than other displayed modes. 20x deformation scale factors were applied to emphasize shape changes of displayed modes.

In conclusion, we demonstrated tunable and selective control of the vibration spectrum of single gold nanoantennas, via the use of carefully placed silica constraint patches. The plasmonic response is rather unaffected if the silica is placed outside of hot spots of electric field enhancement. Such hybrid antennas could in our view form a new platform for the controlled exploration of electromagnetic and acoustic interactions on the nanoscale. 


\section{Associated Content}

Supplementary Information: Analysis of the experimental data. Fabrication details. Experimental set-up. Numerical simulations. Fitting values for the experimental traces. Atomic Force Microscopy profiles for the different nanoresonators. Complementary simulations. Videos showing 3D displacements for both (extensional and breathing modes) for the four types of nanoresonators. This material is available free of charge via the Internet at http://pubs.acs.org.

\section{Author Information}

* Corresponding Authors: e-mail: bragas@df.uba.ar, s.maier@imperial.ac.uk

$\dagger$ These authors contributed equally to this work

$\ddagger$ Present Address: Nonlinear Physics Centre, Research School of Physics and Engineering, The Australian National University, Canberra, ACT 2601, Australia

Note: The authors declare no competing financial interest.

\section{Acknowledgments}

The authors acknowledge funding provided by grants from Universidad de Buenos Aires (20020100100719), the Leverhulme Trust and the Reactive Plasmonics Programme of the U.K. Engineering and Physical Sciences Research Council (EPSRC). R.B. acknowledges the Capes Foundation for a Science Without Borders fellowship (Bolsista da Capes - Proc. n ${ }^{\circ}$ BEX 13.298/13-5). E.C. acknowledge the European Commission for a Marie Curie fellowship. AVB and FDP acknowledge Julian Gargiulo and Fernando Stefani for plasmon resonance measurements.

\section{References}

(1) Young, E. S. K.; Akimov, A. V.; Henini, M.; Eaves, L.; Kent, A. Phys. Rev. Lett. 2012, 108, 226601. 
(2) Moss, D. M.; Akimov, A. V.; Glavin, B. A.; Henini, M.; Kent, A. J. Phys. Rev. Lett. 2011, 106, 066602.

(3) Poyser, C. L.; Akimov, A. V.; Campion, R. P.; Kent, A. J. Sci. Rep. 2015, 5, 8279.

(4) Gadalla, A.; Dehoux, T.; Audoin, B. Planta 2014, 239, 1129-1137.

(5) Dehoux, T.; Audoin, B. J. Appl. Phys. 2012, 112, 124702.

(6) Fong, K. Y.; Poot, M.; Tang, H. X. Nano Lett. 2015, 9, 15, 6116-6120.

(7) Jensen, K.; Kim, K.; Zettl, A. Nat. Nanotechnol. 2008, 3, 533.

(8) Chen, Y. S.; Frey, W.; Kim, S.; Kruizinga, P.; Homan, K.; Emelianov, S. Nano Lett. 2011, 11, 348354.

(9) Dehoux, T; Ghanem, M. A.; Zouani, O. F.; Rampnoux, J. M.; Guillet, Y.; Dilhaire, S.; Durrieu, M.

C.; Audoin, B. Sci. Rep. 2015, 5, 8650.

(10) Hartland, G. V.; Hu, M. Physical Chemistry of Interfaces and Nanomaterials. Edited by Zhang, Jin

Z.; Wang, Zhong L. Proceedings of the SPIE 2002, 4807, 166-176.

(11) Hartland, G. V. Annu. Rev. Phys. Chem. 2006, 57, 403-430.

(12) Tchebotareva, A.; Ruijgrok, P.; Zijlstra, P.; Orrit, M. Laser Photon. Rev. 2010, 4, 581-597.

(13) Major, T. A.; Lo, S. S.; Yu, K.; Hartland, G. V. J. Phys. Chem. Lett. 2014, 5, 866-874.

(14) Tchebotareva, A. L.; van Dijk, M. A.; Ruijgrok, P. V.; Fokkema, V.; Hesselberth, M. H. S.; Lippitz, M.; Orrit, M. Chem. Phys. Chem. 2009, 10, 111-114.

(15) Jais, P. M.; Murray, D. B.; Merlin, R.; Bragas, A. V. Nano Lett. 2011, 11 (9), 3685-3689.

(16) Merlin, R. Solid State Commun. 1997, 102 (2-3), 207-220.

(17) Bartels, A.; Dekorsy, T.; Kurz, H.; Kohler, K. Phys. Rev. Lett. 1999, 82, 1044.

(18) Dekorsy, T.; Cho, G. C.; Kurz, H. Light Scattering in Solids VIII (Springer).Topics in Applied

Physics 2000, 76, 169-209.

(19) Voisin, C.; Del Fatti, N.; Christofilos, D.; Vallee, F. Appl. Surf. Sci. 2000, 164, 131.

(20) Bragas, A. V.; Aku-Leh, C.; Costantino, S.; Ingale, A.; Zhao, J.; Merlin, R. Phys. Rev. B 2004, 69, 205306.

(21) Ruello, P.; Gusev, V. E. Ultrasonics 2015, 56, 21-35.

(22) Brongersma, M. L.; Halas, N. J.; Nordlander, P. Nat. Nanotechnol. 2015, 10, 25-34.

(23) Crut, A.; Maioli, P.; Del Fatti, N.; Vallée, F. Phys. Rep. 2015, 549, 1-43.

(24) O’Brien, K.; Lanzillotti-Kimura, N. D.; Rho, J.; Suchowski, H.; Yin, X.; Zhang, X. Nat. Commun. 2014, 5, 4042 .

(25) Chang, W.-S.; Wen, F.; Chakraborty, D.; Su, M.-N.; Zhang, Y.; Shuang, B.; Nordlander, P.; Sader,

J. E.; Halas, N. J.; Link, S. Nat. Commun. 2015, 6, 7022.

(26) Combe, N; Saviot, L. Phys. Rev. B 2009, 80, 035411.

(27) Wu, B.; Heidelberg, A.; Boland, J. J. Nat. Mater. 2005, 4, 525-529.

(28) Owrutsky, J. C.; Pomfret, M. B.; Brown, D. J. J. Phys. Chem. C 2009, 113, 10947-10955.

(29) Hu, M.; Wang, X.; Hartland, G. V.; Mulvaney, P.; Perez Juste, J.; Sader, J. E. J. Am. Chem. Soc.

2003, 125, 14925-14933.

(30) Hu, M.; Wang, X.; Hartland, G. V.; Salgueiriño-Maceira, V.; Liz-Marzan, L. M. Chem. Phys. Lett. 2003, 372, 767-772.

(31) Zijlstra, P.; Tchebotareva, A. L.; Chon, J. W. M.; Gu, M.; Orrit, M. Nano Lett. 2008, 8 (10), $3493-$ 3497.

(32) Ruijgrok, P. V.; Zijlstra, P.; Tchebotareva, A. L.; Orrit, M. Nano Lett. 2012, 12 (2), 1063-1069.

(33) Marty, R.; Arbouet, A.; Girard, C.; Mlayah, A.; Paillard, V.; Kaixin Lin, V.; Teo, S. L.; Tripathy, S. Nano Lett. 2011, 11 (8), 3301-3306.

(34) Del Fatti, N.; Tzortzakis, S.; Voisin, C.; Flytzanis, C.; Vallee, F. Physica B 1999, 263-264, 54-56. 
(35) Huang, W.; Qian, W.; El-Sayed, M. A. Nano Lett. 2004, 4 (9), 1741-1747.

Table of Contents (graph)

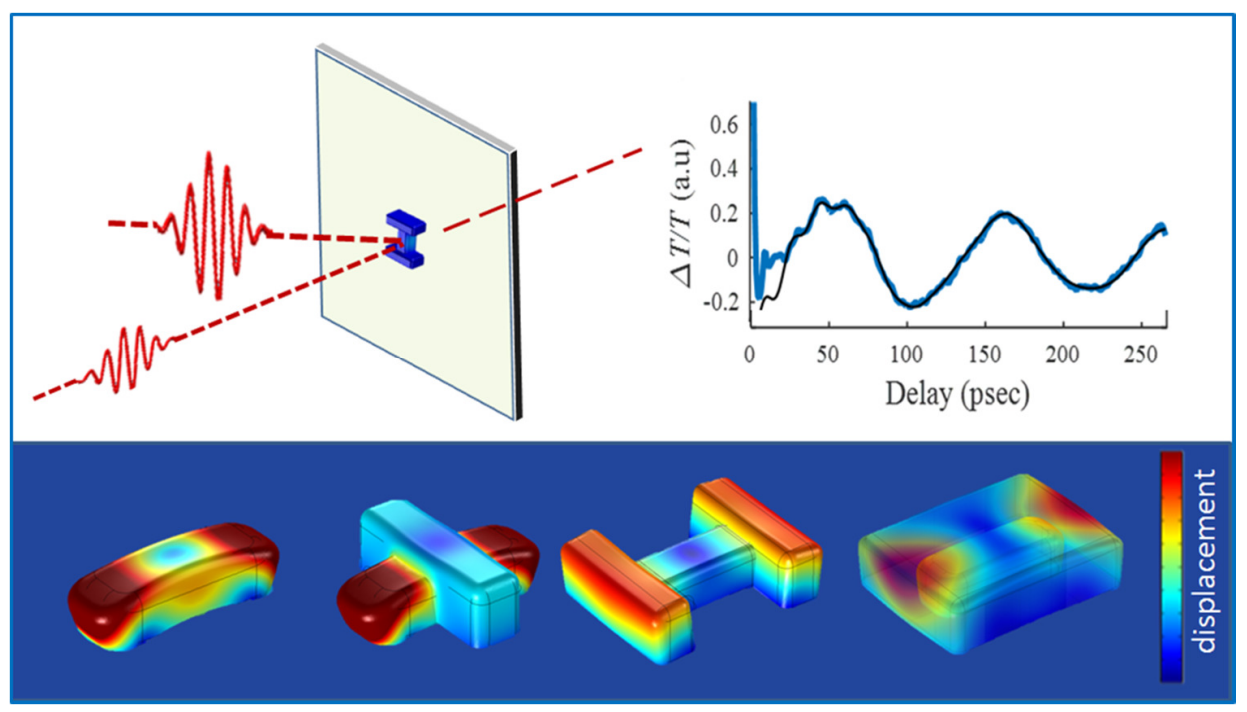

